\section{Disclosure of Positive HIV Status: A Public Health Issue?}

In 1998, the Supreme Court of Canada, in a case in which an HIVinfected man had sex with a woman without divulging his status, ruled that disclosure was necessary where there was a significant risk of HIV transmission. However, significant risk was not quantified, which led to considerable uncertainty in subsequent prosecutions. In 2012, in a comparable case, the Court decided that disclosure was required if there were a realistic risk of HIV transmission. The Court specified that disclosure was unnecessary for vaginal intercourse with a condom when the infected partner had low viral load $(<1500$ copies $/ \mathrm{mL})$. The estimated risk in this scenario is about 1:200,000. Though the Court did not address specifically the issue of anal intercourse, this estimate provides some guidance for other sexual practices.

The reaction to this decision has been strongly divided. Community advocates and organizations rallied uniformly and vigorously against the decision. In contrast, the general population ${ }^{1}$ and a sample of Canadian men who have sex with men (MSM) ${ }^{2}$ generally supported the principle that HIV-positive persons should be legally required to disclose their serostatus to prospective sex partners. The situation has become too acrimonious for open, balanced debate. Persons working in the field have been subjected to both explicit and subtle pressures not to express an opinion supporting the legal sanction of non-disclosure. Public health officials, for fear of being boycotted in their ongoing work on HIV prevention, have generally been silent on this question.

What are the key issues here? Morally, it seems compelling that persons should be provided the relevant information to make an informed decision about consenting to sex. There are also public health implications. Could laws requiring disclosure cause persons to avoid HIV testing since they would be required to disclose if they test positive? On the other hand, the laws would be beneficial if they encouraged HIV-positive persons to disclose their HIV status and/or insist on condom use. The limited data available in Canada to date suggest that the obligation to disclose serostatus does not inhibit MSM from getting tested ${ }^{3}$ and encourages HIV-positive MSM to disclose their serostatus and use condoms. ${ }^{4}$

Some have framed this question as an issue of public versus individual rights, but this is misleading. Public health would favour measures to reduce HIV transmission rates in contrast to the right of an individual to sexual freedom. Nevertheless, the rights of the unknowingly exposed partner are also at play here and in tension with the rights of the HIV-infected person.

This issue continues to be controversial and sensitive. Unfortunately, it has not been debated in or with the public health community because of the strong polarization of opinion. Nonetheless, open mutually respectful and evidence-based debate is badly needed.

\section{Robert Remis \\ Interim Scientific Editor}

\section{REFERENCES}

1. Calzavara L, Allman S, Worthington C, Tyndall M, Adrien A. HIV and AIDS in Canada: A national survey. Available at: http://www.srchiv.ca/

\section{La divulgation de la séropositivité pour le VIH : un problème de santé publique?}

En 1998, dans une cause impliquant un homme infecté par le VIH ayant eu des relations sexuelles avec une femme sans lui divulguer son statut, la Cour suprême du Canada avait jugé que la divulgation était nécessaire en présence d'un risque important de transmission du VIH. Elle n'a toutefois pas quantifié ce risque important, ce qui a entraîné une incertitude considérable dans les poursuites ultérieures. En 2012, dans une cause comparable, la Cour a décidé que la divulgation était nécessaire en présence d'une possibilité réaliste de transmission du VIH. Elle a précisé que la divulgation était inutile en cas de pénétration vaginale avec condom lorsque le partenaire infecté avait une faible charge virale $(<1500$ copies $/ \mathrm{mL})$. Le risque estimatif selon ce scénario est d'environ 1:200 000. Bien que la Cour n'ait pas traité spécifiquement la question de la pénétration anale, cette estimation donne une certaine indication concernant les autres pratiques sexuelles.

La décision de la Cour a fortement divisé l'opinion. Des porteparole et des organismes communautaires l'ont uniformément et vigoureusement contestée. Par contre, le grand public ${ }^{1}$ et un échantillon d'hommes canadiens ayant des relations sexuelles avec des hommes (HARSAH) ${ }^{2}$ ont appuyé dans l'ensemble le principe voulant que les personnes séropositives pour le VIH soient légalement tenues de divulguer leur état sérologique à leurs partenaires sexuels éventuels. La situation s'est envenimée au point de ne plus permettre un débat ouvert et équilibré. Les personnes travaillant sur le terrain subissent des pressions, claires ou subtiles, pour ne pas qu'ils expriment d'opinion à l'appui de la légalité conférée à la nondivulgation. Les autorités de santé publique, de crainte d'être boycottées pour leur travail continu en prévention du VIH, sont généralement silencieuses sur cette question.

Quelles sont les questions clés ici? Moralement, il semble aller de soi que l'on doit fournir aux gens l'information pertinente qui leur permette de prendre une décision éclairée avant de consentir à des relations sexuelles. La question a aussi des répercussions sur la santé publique. Des lois exigeant la divulgation pourraient-elles faire en sorte que des personnes évitent les tests de sérodiagnostic du VIH, car elles seraient alors tenues de divulguer leur séropositivité? En revanche, ces lois seraient bénéfiques si elles encourageaient les personnes séropositives pour le VIH à divulguer leur statut et/ou à insister sur le port du condom. Les données limitées disponibles au Canada jusqu'à maintenant montrent que l'obligation de divulguer l'état sérologique n'empêche pas les HARSAH de se faire tester ${ }^{3}$, et qu'elle encourage les HARSAH séropositifs pour le VIH à divulguer leur état sérologique et à utiliser des condoms ${ }^{4}$.

Certains font de cette question un enjeu opposant les droits collectifs aux droits individuels, mais cette interprétation est trompeuse. La santé publique favoriserait les mesures qui réduisent les taux de transmission du VIH plutôt que le droit d'une personne à la liberté sexuelle. Néanmoins, les droits des partenaires exposés à leur insu sont aussi en jeu ici, et ils ne concordent pas avec les droits des personnes infectées par le VIH.

L'enjeu demeure controversé et délicat. Malheureusement, il n'est pas débattu avec ou dans la communauté de la santé publique en 
NationalSurvey/wp-content/uploads/ 2012/04/Attitudinal-Survey-SummaryReport_June15.pdf (Accessed October 21, 2013).

2. Myers T, Allman D, Adam B, Alexander S, Blais M, Calzavara LM, et al. Male Call Canada Technical Report. Available at: http://www.malecall.ca/ MaleCall/wp-content/uploads/2012/03/Male-Call-Canada-TechnicalReport_13April13_DRAFT.pdf (Accessed October 21, 2013).

3. O'Byrne P, Willmore J, Bryan A, Friedman DS, Hendriks A, Horvath C, et al. Nondisclosure prosecutions and population health outcomes: Examining HIV testing, HIV diagnoses, and the attitudes of men who have sex with men following nondisclosure prosecution media releases in Ottawa, Canada. BMC Public Health 2013;13:94.

4. Kesler M, Kaul R, Loutfy M, Liu J, Brunetta J, Rebbapragada A, et al. Impact of prosecution of non-disclosure of HIV status on attitudes and behavior of HIV-negative and HIV-positive men who have sex with men (MSM) in Toronto, Ontario. 22 $2^{\text {nd }}$ Annual Canadian Conference on HIV/AIDS Research, Vancouver, British Columbia, April 11-14, 2013. Can I Infect Dis 2013;24 (Suppl A):22A-23A (Abstract O044). raison de la forte polarisation de l'opinion. Il n'en demeure pas moins qu'un débat ouvert, mutuellement respectueux et fondé sur les preuves fait cruellement défaut.

\section{Robert Remis \\ Rédacteur scientifique intérimaire}

\section{RÉFÉRENCES}

1. Calzavara, L., S. Allman, C. Worthington, M. Tyndall et A. Adrien. Le VIH et le sida au Canada : une étude nationale, 2012. Sur Internet : http://www.srchiv.ca/NationalSurvey/the-knowledge-bank/publications/ (consulté le 21 octobre 2013).

2. Myers, T., D. Allman, B. Adam, S. Alexander, M. Blais, L.M. Calzavara et coll Male Call Canada Technical Report, 2013. Sur Internet : http://www.malecall.ca/MaleCall/wp-content/uploads/2012/03/ Male-Call-Canada-TechnicalReport_13April13_DRAFT.pdf (consulté le 21 octobre 2013).

3. O'Byrne. P., J. Willmore, A. Bryan, D.S. Friedman, A. Hendriks, C. Horvath et coll. " Nondisclosure prosecutions and population health outcomes: Examining HIV testing, HIV diagnoses, and the attitudes of men who have sex with men following nondisclosure prosecution media releases in Ottawa, Canada ", BMC Public Health, vol. 13 (2013), p. 94

4. Kesler, M., R. Kaul, M. Loutfy, J. Liu, J. Brunetta, A. Rebbapragada et coll. « Impact of prosecution of non-disclosure of HIV status on attitudes and behavior of HIV-negative and HIV-positive men who have sex with men (MSM) in Toronto, Ontario ", $22^{\text {nd }}$ Annual Canadian Conference on HIV/AIDS Research, Vancouver (Colombie-Britannique), 11-14 avril 2013, Can I Infect Dis, vol. 24 (suppl. A) (2013), p. 22A-23A (résumé O044). 